\title{
Identification of Key Pathways and Establishment of a Seven-Gene Prognostic Signature in Cervical Cancer
}

\author{
Ran An, ${ }^{1}$ Silu Meng $\mathbb{D}^{2},{ }^{2}$ and Hua Qian $\mathbb{D}^{1}$ \\ ${ }^{1}$ Department of Dermatology, Children's Hospital of Soochow University, Suzhou, Jiangsu, China \\ ${ }^{2}$ Tongji Medical College, Huazhong University of Science and Technology, Wuhan, Hubei, China \\ Correspondence should be addressed to Silu Meng; lusi_z@hust.edu.cn and Hua Qian; musotan@suda.edu.cn
}

Received 25 September 2021; Revised 18 November 2021; Accepted 14 December 2021; Published 4 February 2022

Academic Editor: Qin Yuan

Copyright (C) 2022 Ran An et al. This is an open access article distributed under the Creative Commons Attribution License, which permits unrestricted use, distribution, and reproduction in any medium, provided the original work is properly cited.

Cervical cancer (CC) remains high morbidity and mortality. We aimed to identify critical pathways underlying cervical carcinogenesis and establish a prognostic signature. Six datasets from the gene expression omnibus (GEO) database were used to screen the differentially expressed genes (DEGs) between CC and normal tissues. We used the unions of the DEGs to perform functional analysis. The 108 overlapped DEGs were analyzed to determine a prognostic signature by Cox regression and Lasso analysis based on The Cancer Genome Atlas (TCGA) database. Gene Set Enrichment Analysis (GSEA) and Immune Cell Abundance Identifier (ImmuCellAI) were used to determine the relationships between the signature and biological functions. The PI3K-Akt signaling pathway, the Ras signaling pathway, and the viral carcinogenesis pathway may be critical for CC development. We identified seven genes (PLOD2, DSG2, SPP1, CXCL8, MCM5, HLTF, and KLF4) to construct a risk score formula. Survival analysis showed that the high-risk group indicated a worse prognosis than the low-risk group $(p<0.0001)$. The AUC of the prognostic signature was 0.7449 , 0.7641 , and 0.8146 at 1,3 , and 5 years. We also identified that the signature is an independent prognostic factor. GSEA showed five pathways were relevant to the signature, such as the adherens junction pathway. The signature also affected the abundances of various types of immune cells, such as B cell, CD4+ T cell, and CD8+ T cell. Further, we found that SPP1 was co-expressed with HK3, CD163, CCL3, CLEC5A, MMP8, TREM1, OLR1, and TREM2. The results of Gene Ontology analysis showed that SPP1 and its co-expressed related proteins mainly affected metabolic process, multicellular organismal process, cell communication, cell proliferation, protein binding, and transporter activity. In conclusion, the present study explored the key pathways for CC development and the seven-gene signature can effectively make the prognosis evaluation of CC patients.

\section{Introduction}

Cervical cancer (CC) is the fourth most commonly diagnosed cancer and the fourth leading cause of cancerassociated mortality in women worldwide, with 570,000 new cases and 311,000 deaths estimated for 2018. The incidence of CC has tended to be younger in recent years [1-4]. The role of human papillomavirus (HPV) infection has been well established $[5,6]$. With the application and development of high-throughput sequencing, many studies have reported many vital genes and pathways in CC carcinogenesis, such as PIK3CA, FBXW7, EP300, MAPK signaling pathway, etc. [7-9]. However, the detailed mechanism of carcinogenesis of CC is still unveiled and needs to be further studied. In clinical practice, early- stage CC patients are mainly treated with surgery, and late-stage patients are treated with chemoradiotherapy [10]. However, the rate of recurrence of CC is approximately $20 \%-25 \%$, and the 5-year survival rate for latestage CC is less than 50\% [11]. The International Federation of Gynecology and Obstetrics (FIGO) stage system has been one of the CC's most important prognostic factors, while the significant differences in survival rate are reported in the same FIGO stage [12]. Therefore, it is crucial to identify sensitive and specific biomarkers that could predict the prognosis of CC patients and monitor patients' outcomes.

Based on the TCGA database and GEO database, we aim to understand the mechanism of $\mathrm{CC}$ carcinogenesis and predict the prognosis of CC patients more precisely. 
Differentially expressed genes (DEGs) were identified using six datasets in the GEO database, and these DEGs were processed to find the key pathways in CC development. Besides, we found out the genes related to the prognosis of patients from among the DEGs and established a model to predict patients' prognosis. To gain further insight into the biological pathways and immune cells changes involved in CC pathogenesis related to this prognostic model, Gene Set Enrichment Analysis (GSEA) and immune cells abundance analysis were performed (Supplementary Figure 1).

SPP1 is a secreted glycophosphoprotein of the SIBLING family [13]. Deregulation of SPP1 has been identified in various cancers [13-15]. Zhao et al. establish a five-gene prognostic model for CC, of which SPP1 is among the genes. The functions of SPP1 include bone metabolism, immune regulation, wound healing, cell survival and tumor progression. In this study, SPP1 may play an important role in the development of cervical cancer. However, its detailed function and mechanism are unclear. We thereforeexplored the function of SPP1 in this study.

\section{Methods}

2.1. Quality Control and DEGs Identification. We recruited six gene expression profiles of CC (GSE6791, GSE63514, GSE7803, GSE9750, GSE39001, and GSE52903) from the gene expression omnibus (GEO) database (https://www. ncbi.nlm.nih.gov/geo) in this study. The information of datasets is shown in Table 1. We downloaded the normalized data of GSE6791, GSE63514, GSE7803, GSE39001, and GSE52903. However, the data of GSE9750 was not normalized, and we normalized its raw data through the R "affy" package with the RMA algorithm method [16]. R "limma" package was used to screen DEGs between normal and CC tissues [17]. Genes with $|\operatorname{LogFC}|>1$ and adjust $p$ value $<0.05$ were considered as DEGs in this study.

2.2. Functional Analysis Based on DEGs. The union of DEGs of the six datasets was processed to Kyoto Encyclopedia of Genes and Genomes (KEGG) and Gene Ontology (GO) analysis by $\mathrm{R}$ "clusterProfiler" package [18]. The $p$ value cutoff and $q$ value cutoff in this study were 0.01 and 0.05 , respectively.

\subsection{Download and Collation of CC Data in TCGA Database.}

The gene expression data of the 304 CC cases (Workflow Type: HTSeq - FPKM-UQ) and corresponding clinical information were downloaded from The Cancer Genome Atlas (TCGA) database (https://portal.gdc.cancer.gov/) by $\mathrm{R}$ "TCGAbiolinks" package [19]. The information of distant metastasis, tumor status, vital status, and overall survival (OS) was from the article of Liu et al. published in Cell in 2018 [20].

2.4. Conduction of the Risk Formula for Prognostic Prediction. The risk score formula was constructed using the data of CC of the TCGA database. First, we used the Venn diagram to find the overlapped DEGs of the six datasets and finally got
TABLE 1: Information of the datasets used in this study.

\begin{tabular}{lcccc}
\hline \multirow{2}{*}{ Datasets } & \multirow{2}{*}{ Year } & \multirow{2}{*}{ Platform } & \multicolumn{2}{c}{ Participants } \\
& & & Normal & Cervical cancer \\
\hline GSE6791 & 2007 & GPL570 & 8 & 20 \\
GSE63514 & 2015 & GPL570 & 24 & 28 \\
GSE7803 & 2007 & GPL96 & 10 & 21 \\
GSE9750 & 2008 & GPL96 & 24 & 33 \\
GSE39001 & 2013 & GPL6244 & 5 & 19 \\
GSE52903 & 2015 & GPL6244 & 17 & 55 \\
\hline
\end{tabular}

108 DEGs. By performing univariate Cox regression analysis with $\mathrm{R}$ "survival" package, the association between the expression of 108 DEGs and patient's OS was assessed. 26 DEGs with a $p$ value of less than 0.05 were included in the subsequent analysis. Second, based on the above identification of prognosis-related genes for CC, we further needed to narrow the gene range and establish a prognostic signature. Thus, we performed the Least absolute shrinkage and selection operator (Lasso) analysis by R "glmnet" package, which constructs a more refined model using a penalty function. This method can reduce the model's complexity and reduce the weight of some unimportant indicators to 0 , and 13 DEGs were left in this step. Next, we used the 13 DEGs to perform a multivariate Cox regression analysis. In this step, we further optimized the model based on the Akaike's Information Criterion (AIC) value, and finally 7 genes (Procollagen-Lysine, 2-Oxoglutarate 5-Dioxygenase 2 (PLOD2), Desmoglein 2 (DSG2), Secreted Phosphoprotein 1 (SPP1), C-X-C Motif Chemokine Ligand 8 (CXCL8), Minichromosome Maintenance Complex Component 5 (MCM5), Helicase Like Transcription Factor (HLTF), Kruppel Like Factor 4 (KLF4)) were left. A risk formula (prognostic signature) was then established based on a linear combination of these DEGs' expression levels, weighted by their regression coefficients derived from the multivariate Cox regression model. The formula was as follows: risk score $=[$ Expression level of PLOD2 $*(0.252751)]+[\mathrm{Ex}-$ pression level of DSG2 * (0.381041)] + [Expression level of SPP1 $*(0.170055)]+[$ Expression level of CXCL8 $*$ $(0.163175)]+[$ Expression level of MCM5 * $(-0.514752)]+[$ Expression level of HLTF $*(-0.245823)]$ + [Expression level of KLF4 $*(-0.184198)]$. Finally, a risk score was computed for each patient with this formula and patients were classified into high-risk and low-risk group, by taking the median risk score as a cutoff point. To detect the seven genes function, COXPREdb (https://coxpresdb.jp/) was used to find the top 200 co-expressed genes of the seven genes and then these co-expressed were processed to KEGG analysis by R "clusterprofiler" package [18].

2.5. Assessment of the Signature's Effect. We ranked each patient's risk score and counted the number of deaths in the high-risk group and the low-risk group. Kaplan-Meier estimate based on the log-rank test was used to compare the survival difference between the high-risk group and the lowrisk group by $\mathrm{R}$ "survminer" package. Receiver operating characteristic (ROC) curves were employed to compare the 
sensitivity and specificity of the survival prediction based on the risk score model by R "survivalROC" package. To test whether the prognostic signature was independent of clinical variables, we performed the univariate Cox regression analysis, and variables with $p$ value $<0.05$ were then analyzed by multivariable Cox regression analysis. Then the independent prognostic factor was chosen to construct the nomogram by using R packages "rms" and "forestplot," and the ROC curves of the predictive nomogram were also performed. All statistical analyses were finished with $\mathrm{R}$ version 3.6.3.

2.6. Gene Set Enrichment Analysis (GSEA). GSEA (version 4.1.0) was used to explore the signaling pathways related to the risk score model [21]. The phenotypes were determined by the cutoff value of the risk score. The annotated gene set was selected (c2.cp.kegg.v7.1.symbols.gmt) as the reference gene set. Gene set permutations were performed 1000 times for analysis. The normalized enrichment score (NES), nominal $p$ value, and false discovery rate (FDR) $q$ value were used to sort the pathways enriched in each group. Pathways with NES $>1$, nominal $p$ value $<0.05$ and FDR $q$ value $<0.25$ were selected out.

2.7. Immune Cell Component Analysis. CXCL8 is an immune-related gene, and we therefore used the Immune Cell Abundance Identifier (ImmuCellAI), which estimates the abundance of 24 immune cells, to detect whether the prognostic signature will affect the immune microenvironment [22].

2.8. Linkedomics Database Analysis. We further analyzed the cancer-promoting mechanism of SPP1 by using LinkedOmics analysis software based on the TCGA database. The co-expression-related proteins of SPP1 were searched using LinkedOmics database. Cancer types to be studied were directly selected (38 cancers in total). Cervical cancer was selected as an example in this study. Analysis was carried out according to molecular typing, staging, and other data. The statistical analysis method was person correlation coefficient analysis. Then, GO analysis was performed on SPP1 and its co-expressed related proteins.

\section{Results}

3.1. Quality Control and DEGs Identification. We selected six gene expression datasets of CC (GSE6791, GSE63514, GSE7803, GSE9750, GSE39001, and GSE52903) to assess the DEGs between CC and normal tissues. The quality control results of the six datasets are shown in Supplementary Figure 2. The information of the datasets and the number of DEGs of each dataset are shown in Table 1 and Figure 1(a).

3.2. Functional Analysis Based on DEGs. The union of DEGs of the six datasets was processed to KEGG and GO analysis. In the KEGG analysis, the upregulated genes enriched in many well-known pathways, such as the cell cycle, the DNA replication, and the nucleotide excision repair (Figure 1(b)). And the downregulated genes are related to many cancerrelated pathways, such as the Ras signaling pathway and the PI3K-Akt signaling pathway (Figure 1(b)). In the GO analysis, the upregulated genes mainly participate in the biological process (BP) related to cell cycle, such as the DNA replication, the $\mathrm{G} 2 / \mathrm{M}$ transition of mitotic cell cycle, the regulation of mitotic cell cycle phase transition, etc. (Figure 1(c)). While the downregulated genes mainly enrich in BP of the cornification, the epidermis development, the peptide cross-linking, etc. (Figure 1(d)). The results of cellular component (CC) and molecular function (MF) are also shown in Figures 1(c) and 1(d).

3.3. Information of CC in the TCGA Database. We included and counted the CC's clinical information, including age, keratinization, FIGO stage, differentiation, and lymphovascular invasion. And the information of distant metastasis, tumor status, vital status, and OS information was from the article Liu et al. (Table 2) [20]. Also, the gene expression data and clinical information were matched, which were used for the subsequent survival analysis.

3.4. Establishment of a Seven-Genes Signature for Prognosis Prediction. The Venn diagram shows the 108 overlapped DEGs of the six datasets, of which 69 were upregulated genes and 39 were downregulated genes (Figure 2(a)). The names of the 108 DEGs are shown in the Supplementary Table 1. The relationship between the 108 DEGs and the patient's OS was assessed by univariate Cox regression analysis, and 26 DEGs whose parameter $p$ values were less than 0.05 were chosen for subsequent analysis (Supplementary Table 2). Considering the number of genes and collinearity, Lasso regression analysis was used to narrow the gene range, and only 13 genes were remained in this step (Figures 2(b) and 2(c)). Next, the 13 genes were processed to the multivariate Cox regression model, and we further optimized the model based on the AIC value. Finally, 7 genes (PLOD2, DSG2, SPP1, CXCL8, MCM5, HLTF, KLF4) were screened out as the predictor signature and their detailed information including coefficients, $\mathrm{HR}$ value, and $p$ value is shown in Figure 2(d). Of these, positive coefficients for the PLOD2, DSG2, SPP1, and CXCL8 indicated that their upregulated levels of expression were associated with shorter survival. The negative coefficient of MCM5, HLTF, and KLF4 indicated that upregulated level of expression was associated with longer survival. A prognostic model based on the coefficients was established and the risk score formula was as follows: risk score $=[$ Expression level of PLOD2 * $(0.252751)]+[$ Expression level of DSG2 $*(0.381041)]$ $+[$ Expression level of SPP1 $*(0.170055)]+[$ Expression level of CXCL8 $*(0.163175)]+[$ Expression level of MCM5 $*(-0.514752)]+[$ Expression level of HLTF * $(-0.245823)]$ $+[$ Expression level of KLF4 $*(-0.184198)]$. We then calculated the seven-genes signature risk score of each patient in using the above formula. The median risk score (0.3865) was used as the cutoff point to divide the patients into two groups, the high-risk group $(N=152)$, and the low-risk 

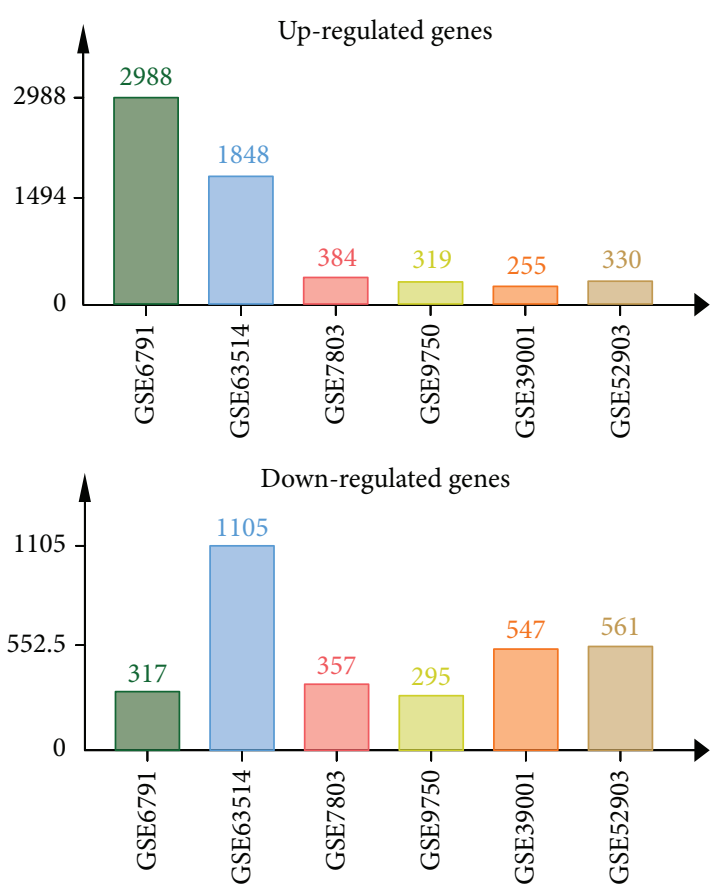

(a)

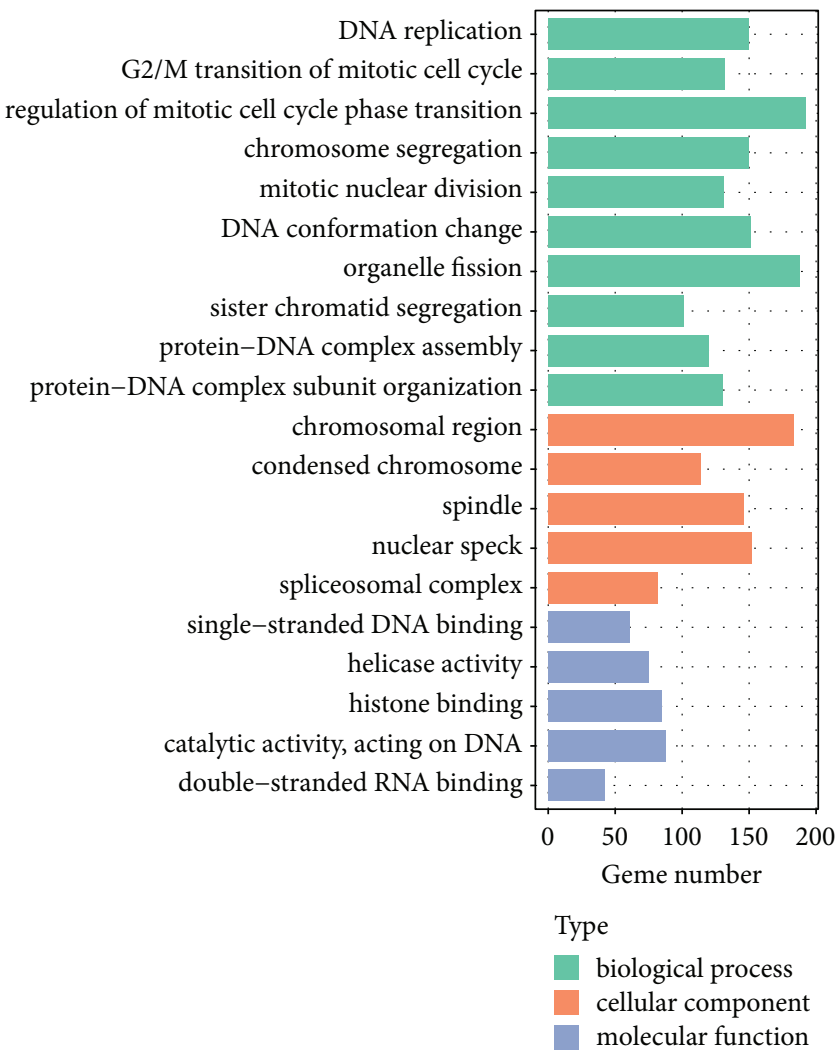

(c)

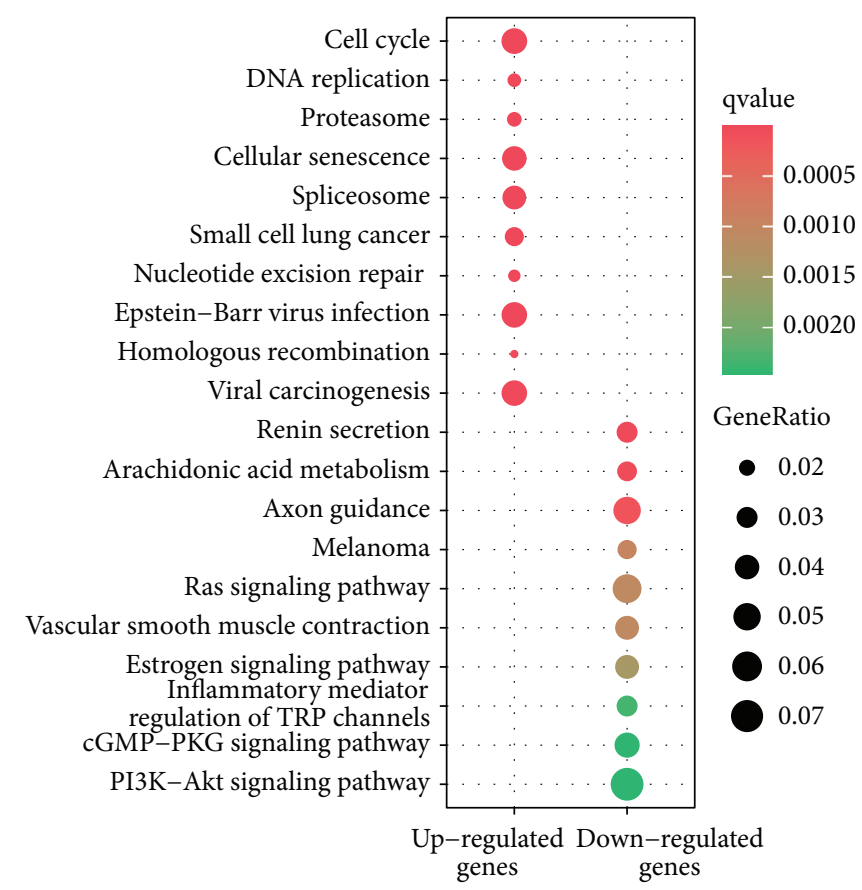

(b)

cornification
epidermis development
peptide cross-linking

unsaturated fatty acid metabolic process

hormone metabolic process epithelial cell proliferation

multicellular organismal homeostasis positive regulation of protein kinase B signaling positive regulation of epithelial cell proliferation extracellular matrix organization cornified envelope collagen-containing extracellular matrix apical part of cell cell-cell junction apical plasma membrane extracellular matrix structural constituent structural constituent of epidermis heparin binding transmembrane receptor protein tyrosine kinase activity transmembrane receptor protein kinase activity

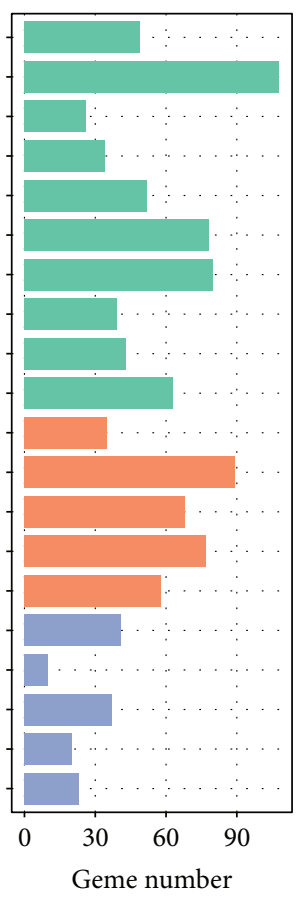

Type

biological process cellular component molecular function

(d)

FIGURE 1: The number of DEGs in each dataset and KEGG and GO analysis of DEGs. (a) The number of DEGs in each dataset. (b) KEGG analysis. (c) GO analysis of upregulated genes. (d) GO analysis of downregulated genes. DEGs, differentially expressed genes; KEGG, Kyoto Encyclopedia of Genes and Genomes; GO, Gene Ontology.

group $(N=152)$ (Supplementary Table 3$)$. In addition, to understand the function of the seven genes, the co-expressed genes were processed to KEGG analysis and they enriched in many cancer-related pathways, such as the PI3K-Akt signaling pathway, the Toll-like receptor signaling pathway, and the P53 signaling pathway. And the co-expression genes 
TABLE 2: Characteristics of the cervical cancer patients in the TCGA database.

\begin{tabular}{|c|c|c|c|}
\hline Clinical characteristics subgroup & & Frequency & Percentage \\
\hline Total & & 304 & \\
\hline Age & Range: $20-88$ (average: 48.2 , median: 46 ) & & \\
\hline \multirow{3}{*}{ Keratinization } & No & 119 & 68.4 \\
\hline & Yes & 55 & 31.6 \\
\hline & $\mathrm{N} 1$ & 60 & 31.1 \\
\hline \multirow{4}{*}{ FIGO } & I & 162 & 54.5 \\
\hline & II & 69 & 23.2 \\
\hline & III & 45 & 15.2 \\
\hline & IV & 21 & 7.1 \\
\hline \multirow{2}{*}{ Differentiation grade } & $\leq \mathrm{G} 2$ & 153 & 56.3 \\
\hline & $\geq \mathrm{G} 3$ & 119 & 43.7 \\
\hline \multirow{2}{*}{ Lymphovascular invasion } & Absent & 71 & 47.3 \\
\hline & Present & 79 & 52.7 \\
\hline \multirow{2}{*}{ Distant metastasis } & No & 273 & 89.8 \\
\hline & Yes & 31 & 10.2 \\
\hline \multirow{2}{*}{ Tumor status } & Tumor free & 197 & 71.1 \\
\hline & With tumor & 80 & 28.9 \\
\hline \multirow{2}{*}{ Vital status } & Alive & 233 & 76.6 \\
\hline & Dead & 71 & 23.4 \\
\hline
\end{tabular}

of PLOD2 and DSG2 also enriched in the Human papillomavirus infection which is inseparable with CC development (Supplementary Figure 3).

3.5. Assessment of the Signature's Effect. The samples were ranked according to their risk scores (Figure 3(a)) and the number of deaths increased significantly with the increase of the risk score (Figure 3(b)). A heatmap was visualized to demonstrate the expression profiles of the 7 genes (Figure 3(c)). We identified that the OS of the high-risk group is significantly shorter than the low-risk group by Kaplan-Meier method (Figure 3(d)). To further investigate the discrimination power of the signature, ROC curves were further performed. The area under the curve (AUC) of the signature was $0.7449,0.7641$, and 0.8146 at 1,3 , and 5 years (Figure 3(e)). To test whether the signature was independent of clinical variables, univariate and multivariate Cox regression analysis were performed, showing that lymphovascular invasion (HR, 7.0050; 95\%CI, 1.3995-35.0632; $p=0.0178)$, tumor status (HR, 94.2939; 95\%CI, 17.2409-515.7118; $p<0.0001)$, and the signature (HR, 6.5823 ; 95\%CI, $1.9382-22.3537 ; p=0.0025)$ are the independent predictor of poor overall survival (Figures 4(a) and 4(b)). Next, we established a nomogram combining the three independent factor (Figure 4(c)). The AUC for 1-, 3-, and 5year survival using the predictive nomogram reached 0.7549 , 0.8062 , and 0.8064 , respectively (Figure $4(\mathrm{~d})$ ).

3.6. Identification of the Seven-Gene Signature Altered Pathways. To identify potentially altered signaling pathways related to the signature, we performed GSEA analysis based on the risk score classification. We selected out 5 significantly enriched signaling pathways based on the standard NES $>1$, nominal $p$ value $<0.05$, and FDR $q$ value
$<0.25$, including adherent junction, ECM-receptor interaction and focal adhesion, etc. (Figure 5 and Supplementary Table 4).

3.7. Immune Cell Component Analysis. ImmuCellAI was used to analyze the difference of immune cells between the high-risk and the low-risk group (Supplementary Table 5). The abundance of Tr 1 cells, B cells, CD4+ T cells, and CD8+ $\mathrm{T}$ cells were significantly increased in the low-risk group, while the Th17 cells, NKT cells, NK cells, and Neutrophil were significantly increased in the high-risk group (Figure 6).

3.8. Analysis of SPP1 Co-Expressed Related Proteins and Gene Ontology. We further analyzed the cancer-promoting mechanism of SPP1 by using LinkedOmics (https://www. linkedomics.org/login.php) analysis software based on the TCGA database (Figure 7(a)). Through analysis, we found that SPP1 was co-expressed with HK3, CD163, CCL3, CLEC5A, MMP8, TREM1, OLR1, and TREM2 (Figure 7(b)). The results of GO analysis showed that SPP1 and its coexpressed related proteins mainly affected metabolic process, multicellular organismal process, cell communication, cell proliferation, protein binding, and transporter activity (Figure 7(c)). These results further elucidate the carcinogenic mechanism of SPP1, which increased the understanding of SPP1.

\section{Discussion}

The morbidity and mortality of CC remain high. Although the role of HPV in CC has been well established and many researches have been done in uncovering the potential molecular mechanism of CC development, the underlying 
Up-regulated genes

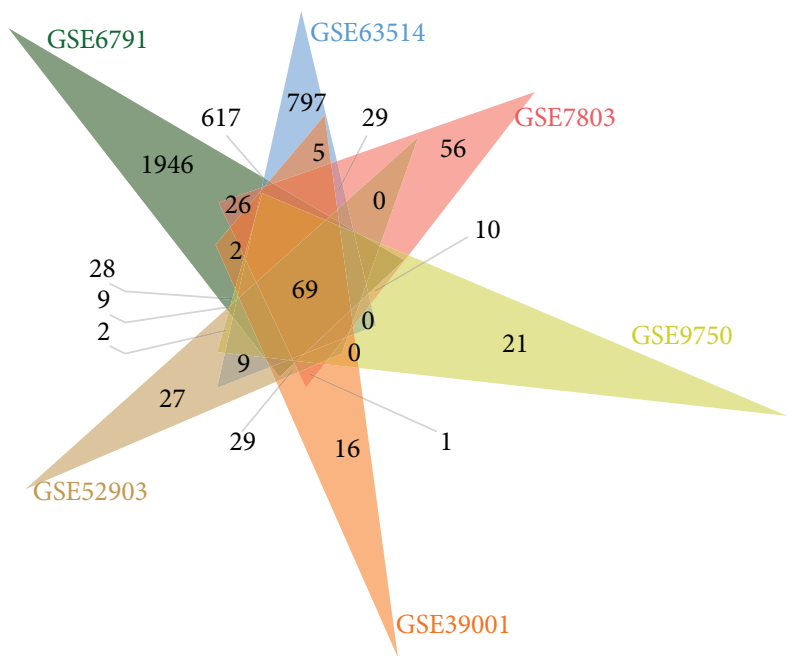

Down-regulated genes

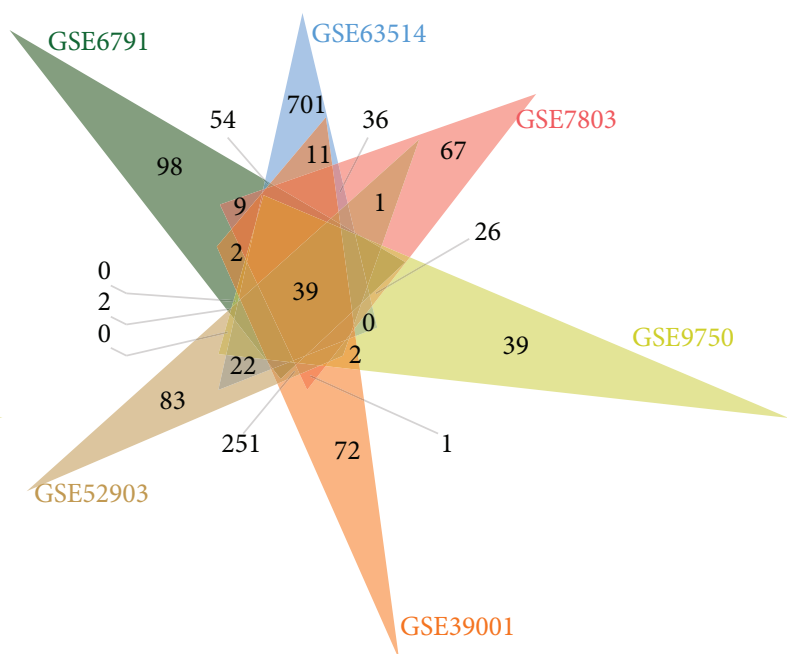

(a)

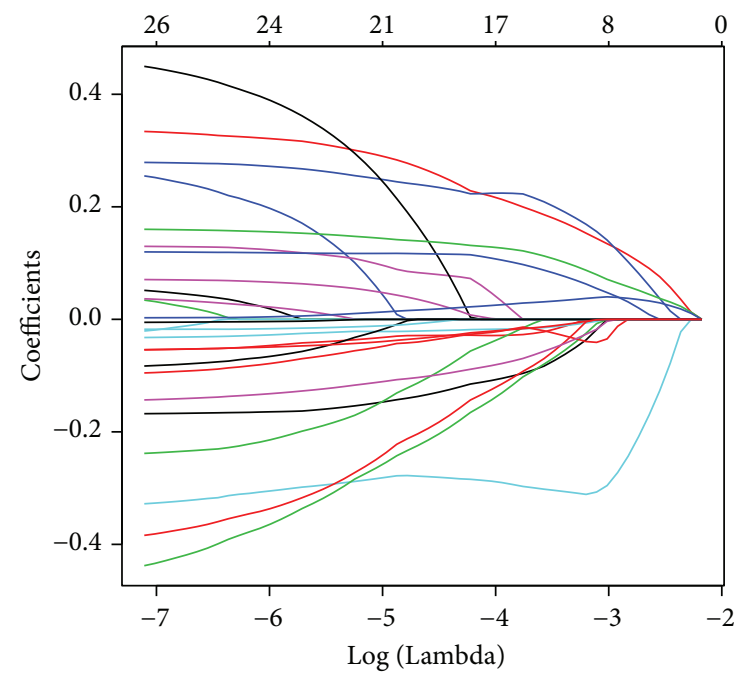

(b)

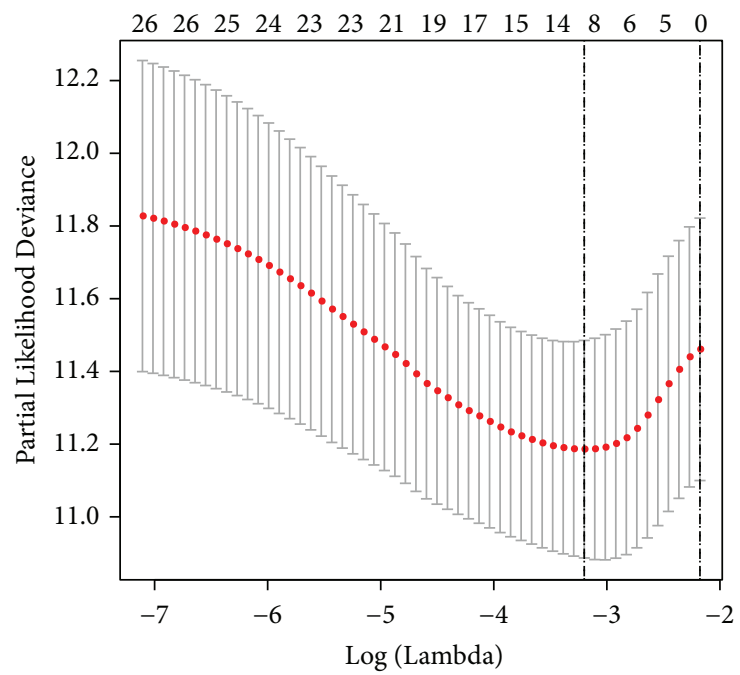

(c)

FIgUre 2: Continued. 


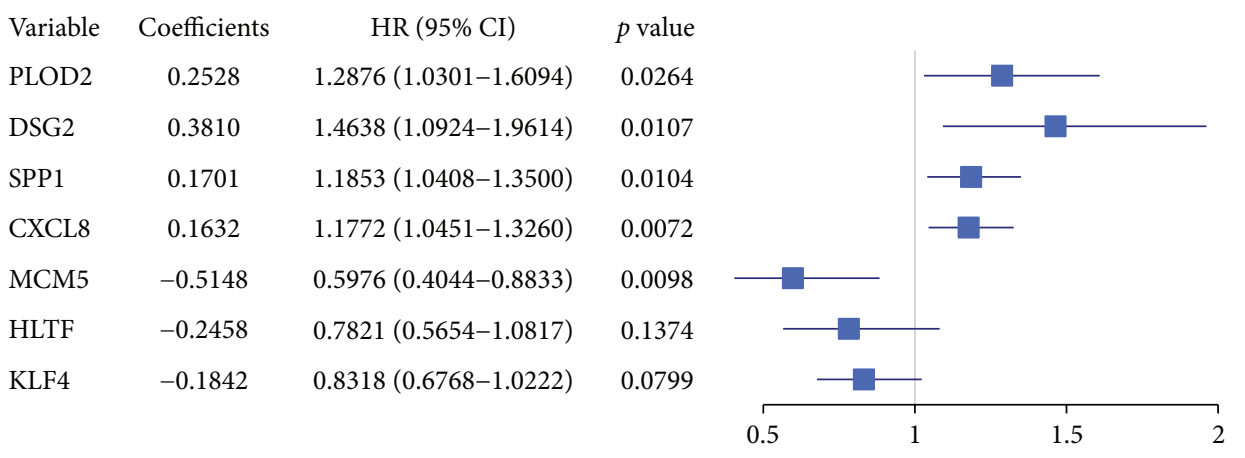

(d)

Figure 2: Construction of the seven-gene prognostic signature for cervical cancer. (a) The overlapped upregulated genes. (b) The overlapped downregulated genes. (c) The lasso analysis identified the most correlated genes with prognosis. (d) The distribution of each lambda and confidence interval. (e) Further narrowing of the gene range by multivariate Cox analysis and 7 genes was determined. Lasso, least absolute shrinkage and selection operator.

mechanism remains unclear. The surgical and chemoradiotherapy of CC are very mature, while many patients still suffered from recurrence and metastasis. The present research performed an integrated bioinformatical analysis based upon six mRNA expression profile datasets of the GEO database and 304 CC patients of the TCGA database to further uncover the mechanism of CC and discover more promising and valuable prognosis-related biomarkers in CC.

We used the union of the DEGs of the six datasets to perform KEGG and GO analysis. In the KEGG analysis, the upregulated genes enriched in the cell cycle, the DNA replication, the nucleotide excision repair, etc., and the DEGs may promote cell proliferation by being involved in these pathways. These pathways are common in various kinds of cancer, such as colorectal cancer and gastric cancer [23-25]. In addition, the upregulated genes also enriched in the viral carcinogenesis, which may also prove the role of HPV in the development of CC. The downregulated genes enriched in the PI3K-Akt signaling pathway and the Ras signaling pathway, which have been reported to play critical roles in CC [9]. In the GO analysis, the upregulated genes were mainly related to cell cycle related terms, such as the DNA replication, the G2/M transition of mitotic cell cycle, and the regulation of mitotic cell cycle phase transition. This result is similar to that of KEGG to a large extent. In contrast, the downregulated genes enriched in many epidermis-related pathways, such as epidermis development, epithelial cell proliferation, positive regulation of epithelial cell proliferation, and structural constituent of epidermis. In summary, the enriched GO terms and KEGG pathways explained the specific molecular mechanisms of CC to some extent.

We identified 108 common DEGs between the CC tissues and normal tissues of the six datasets, including 69 upregulated genes and 39 downregulated genes. We further analyzed the relationship between the prognosis of CC patients and the expression levels of the 108 DEGs by univariate Cox analysis, of which 26 DEGs indicated the significant correlation with OS $(p<0.05)$. Next, we performed both lasso and multivariate Cox analysis to narrow the gene range, and finally we got 7 genes (PLOD2, DSG2, SPP1, CXCL8, MCM5, HLTF, KLF4) to establish a prognostic model which was able to distinguish CC patients into the high-risk group and low-risk group. PLOD2 encodes the key enzyme mediating the formation of the stabilized collagen cross-link, which sometimes can be considered as the "highway" for cancer cell migration and invasion [26]. PLOD2 overexpressed in many cancers, including hepatocellular carcinoma, breast cancer, and sarcoma [26]. Overexpression of PLOD2 can also induce cell migration and invasion in CC and PLOD2 is correlated with the prognosis of CC patients [27, 28]. DSG2 is a cell adhesion protein of the cadherin superfamily, which can regulate cellcell contact. And DSG2 has been reported to play key roles in tumorigenesis $[29,30]$. Qin et al. have reported that DSG2 can promote tumor proliferation and metastasis and is correlated with poor prognosis in early-stage CC [31]. CXCL8 is an important cytokine that can modulate proliferation, invasion, and migration of tumor cells and can induce tumor immunosuppression. It has been reported that the CXCL8-CXCR1/2 axis has the potential to be applied as a cancer therapeutic target [32]. Yan et al. found that CXCL8 high expression was a poor independent prognostic parameter for CC patients [33]. MCM5 is a member of the MCM family of chromatin-binding proteins and participates in cell cycle regulation [34]. MCM5 has been reported as a predictive biomarker for both cervical preinvasive neoplasia and CC [35]. HLTF belongs to the SWI/SNF family of proteins involved in chromatin remodeling and DNA repair, suggesting that it acts as a tumor suppressor gene [36]. HLTF expression is altered in cancer through two mechanisms: gene silencing by promoter hypermethylation or expression of truncated proteins that lack functional domains [36]. These mechanisms have been widely proved in digestive tract cancers $[37,38]$. In CC, Cho et al. showed overexpression of HLTF might confer patients with resistance to radiation [39]. KLF4 functions both as a tumor suppressor and an oncogene, which is involved in cell differentiation and cell-cycle arrest [40]. It has shown that KLF4 can regulate cell proliferation, migration, and invasion in 


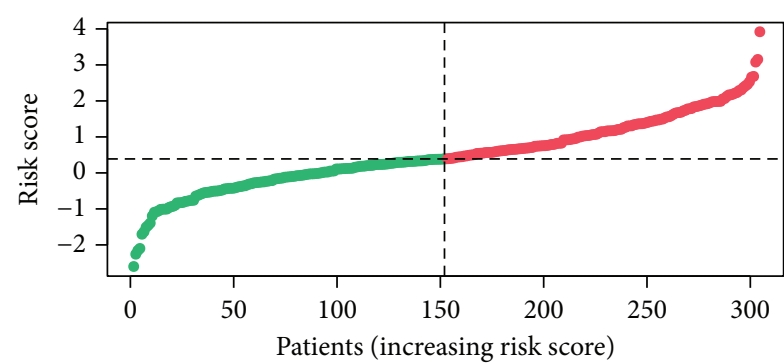

- High risk

- Low risk

(a)

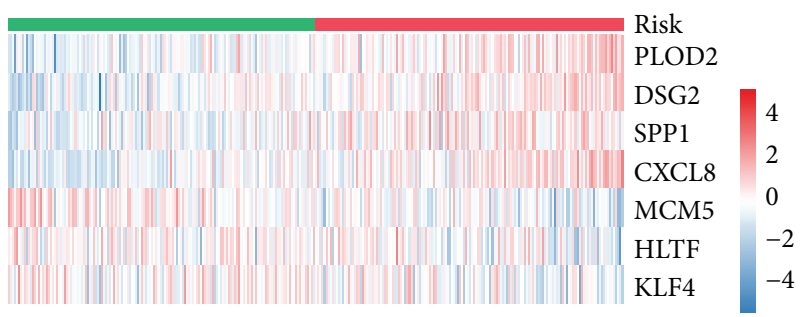

Risk

- Low risk

- High risk

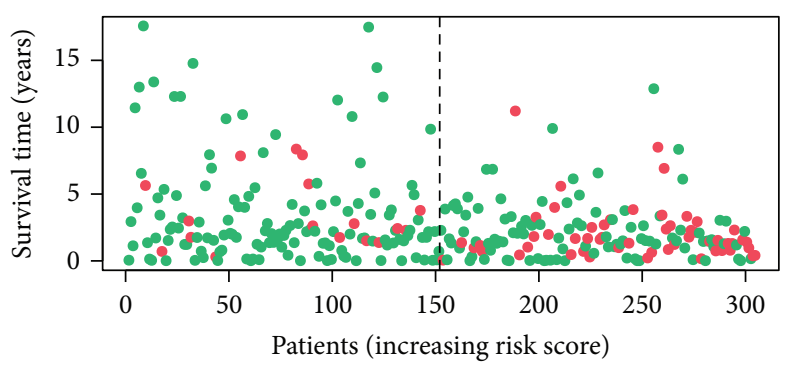

- Dead

- Alive

(b)

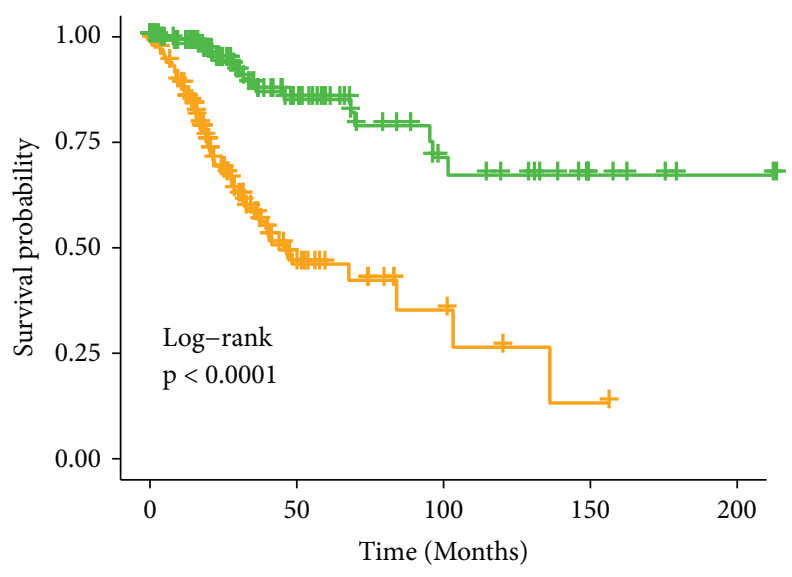

+ High risk

+ Low risk

(c)

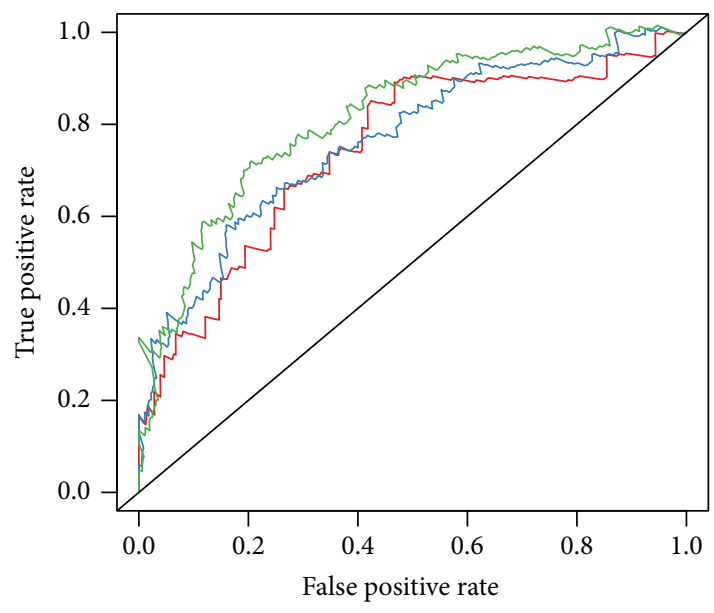

(d)

1-year survival $(\mathrm{AUC}=0.7449)$
3-year survival $(\mathrm{AUC}=0.7641)$
5-year survival $(\mathrm{AUC}=0.8146)$

(e)

Figure 3: Assessment of the prognostic signature. (a) Distribution of risk score in the high-risk group and the low-risk group. (b) Survival status between the high-risk group and the low-risk group. (c) The heatmap of expression profile of 7 genes. Red parts represent upregulation, blue parts represent downregulation, and white parts represent no differential expression. (d) Survival analysis showed that the patients in the high-risk group had statistically significantly worse overall survival than those in low-risk group based on TCGA database. (e) ROC analysis was performed to calculate the AUC of 1-, 3-, and 5-year survival for this prognostic signature. TCGA, The Cancer Genome Atlas; ROC, receiver operating characteristic; AUC, area under the curve. 


$\begin{array}{lcc}\text { Variable } & \text { HR }(95 \% \mathrm{CI}) & p \text { value } \\ \text { Age } & 1.0171(0.9995-1.0349) & 0.0570 \\ \text { Keratinization } & 1.6856(0.8725-3.2566) & 0.1202 \\ \text { FIGO } & 1.4916(1.1982-1.8569) & 0.0003 \\ \text { Grade } & 0.8894(0.5267-1.5018) & 0.6610 \\ \text { Lymphovascular invasion } & 10.0413(2.3613-42.7000) & 0.0018 \\ \text { Distant metastasis } & 3.1412(1.8657-5.2886) & <0.0001 \\ \text { Tumor status } & 21.5956(11.2868-41.3202) & <0.0001 \\ \text { Risk } & 4.7053(2.7001-8.1997) & <0.0001\end{array}$

(a)

Variable

FIGO

Lymphovascular invasion

Distant metastasis

Tumor status

Risk
HR (95\% CI)

1.2897 (0.7273-2.2872)

$7.0050(1.3995-35.0632)$

$0.3384(0.1139-1.0059)$

94.2939 (17.2409-515.7118)

$6.5823(1.9382-22.3537)$
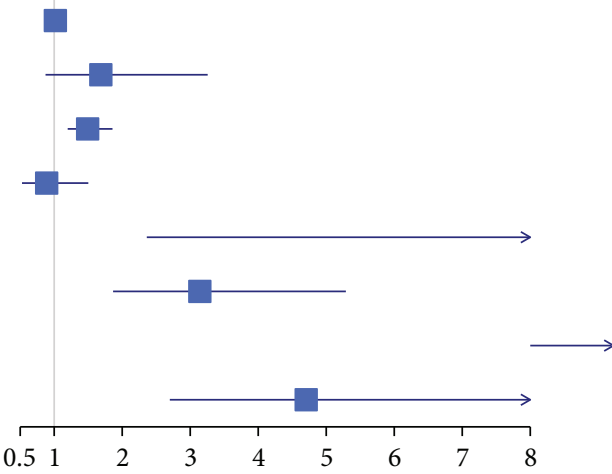

$p$ value

0.384

0.0178

0.0512

$<0.0001$

0.0025

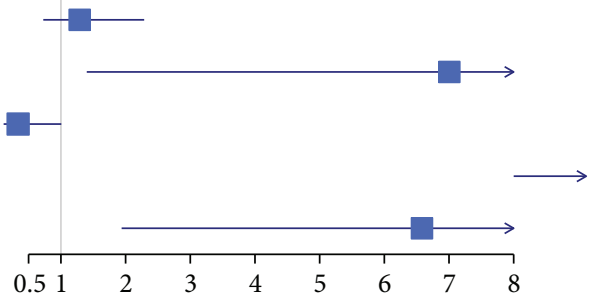

(b)

Points

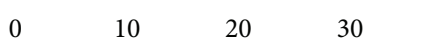

Yes

Lymphovascular invasion No

Tumor status

Tumor free

With tumor

Risk

Low risk

High risk

Total Points

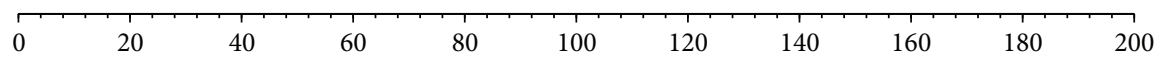

1 - year survival

$$
0.99
$$

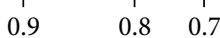

3 - year survival

5 - year survival

0.99

(c)

FIgUre 4: Continued. 


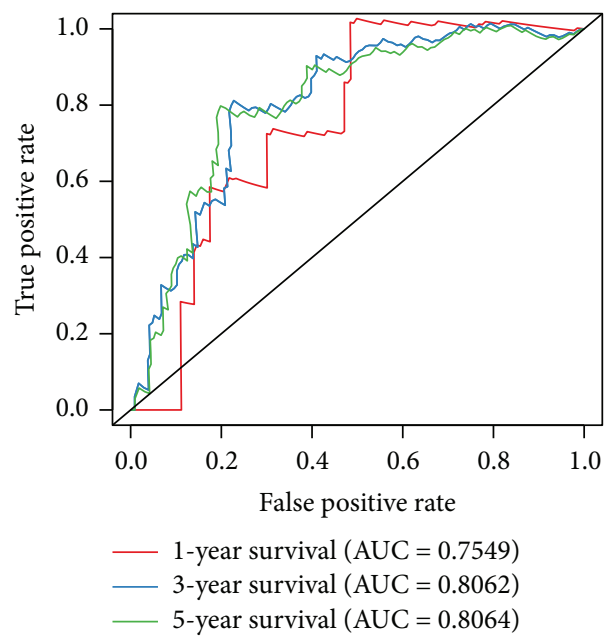

(d)

Figure 4: The Cox regression analysis for evaluating the independent prognostic value of the risk score. (a) The univariate Cox analysis. (b) The multivariate Cox analysis. (c) The nomogram to predict the probabilities 1-year, 3-year, and 5-year OS in patients. (d) ROC curves according to the nomogram. Age, continuous variable; keratinization, yes vs. no; FIGO, continuous variable at I, II, III, and IV stages; grade, $\geq \mathrm{G} 3$ vs. $<\mathrm{G} 3$; lymphovascular invasion, yes vs. no; distant metastasis, yes vs. no; tumor status, with tumor vs. tumor free; ROC, receiver operating characteristic.

multiple cancers, including breast cancer, gastrointestinal cancer, and esophageal cancer [41-43]. Yang and Zheng identified the tumor suppressor role of KLF4 in CC and found that promoter hypermethylation of KLF4 can inactive its tumor suppressor function in CC $[44,45]$. To further understand the function of the 7 genes we selected, the top 200 co-expressed genes of the 7 genes were processed to KEGG analysis, which shows many critical pathways for CC development, including the PI3K-Akt signaling pathway, the Huam papillomavirus infection, and the p53 signaling pathway.

Based on the prognostic signature, the number of deaths increased significantly with risk score increase. Kaplan-Meier survival analysis showed that the high-risk group indicated a worse prognosis than the low-risk group $(p<0.0001)$. The AUC of the risk model was 0.7449, 0.7641, and 0.8146 at 1,3 , and 5 years. Next, the univariate and multivariate Cox regression analyses were performed, identifying that lymphovascular invasion, the tumor status, and the signature are independent prognostic factors in CC. The AUC of the predictive nomogram combining the three independent factors for 1, 3, and 5-year survival reached $0.7549,0.8062$, and 0.8064 .

To further investigate the model's underlying mechanism, we performed the GSEA to explore the signaling pathways related to the risk score model, and 5 pathways were selected out. The adherens junction pathway is an element of cell-cell junction, essential for maintaining tissue architecture and cell polarity and can regulate cell movement and proliferation. E-cadherin, and $\alpha$ - and $\beta$-catenin are the main components of AJ [46]. Loss or downregulation of E-cadherin expression is frequently observed in cancers and correlates with the malignancy of the tumors [47]. Fujimoto et al. have reported that decreased expression of main adhesion molecules may result in invasiveness and metastasis of CC [48]. Li et al. identified that the genes at breakpoints of HPV integration in CC also enrich in the adherens junction pathway [49]. The ECM-receptor interaction pathway controls many cellular activities such as adhesion, migration, differentiation, proliferation, and apoptosis [50]. This pathway participates in the progression of various cancers such as breast cancer, prostate cancer, and gastric cancer [51-53]. Using two datasets of the GEO database, $\mathrm{Wu}$ et al. found that the ECM-receptor interaction pathway is the key pathway during CC development [54]. Focal adhesion refers to the specialized structures at cellextracellular matrix contact points, which play essential roles in important biological processes, including cell motility, cell proliferation, and cell differentiation [55]. Increased expression and amplification of the focal adhesion kinase gene in human cancer cells are common [56]. Xu et al. showed that PLOD2 could improve the migration and invasion of CC cells by focal adhesion formation [27]. We also found the other two pathways in this study, the renal cell carcinoma and small cell lung cancer pathways, which may indicate that the underlying molecular mechanism of CC development may coincide with the two kinds of cancer. In addition, we analyzed whether the risk model affects the immune microenvironment. The abundance of $\operatorname{Tr} 1$ cells, B cells, CD4+ $\mathrm{T}$ cells, and CD8+ T cells are significantly increased in the low-risk group, while the Th17 cells, NKT cells, NK cells, and Neutrophil are significantly increased in the high-risk group. Wang et al. found that a higher level of activated memory CD4+ T cells was independently associated with favorable OS in CC [57]. Although we don't have the result of activated memory CD4+ T cells, the abundance of CD4+ T cells is higher in the low-risk group. It has shown that both the CC patients and cervical precancerous lesion patients have a 

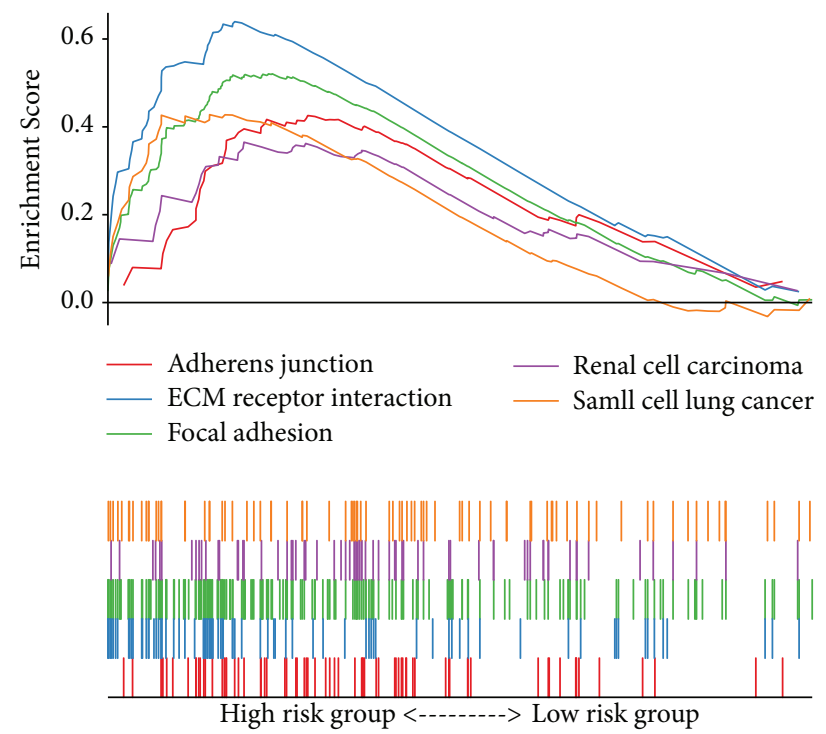

FIGURE 5: A merged enrichment plot from gene set enrichment analysis (GSEA) including enrichment score and gene sets.

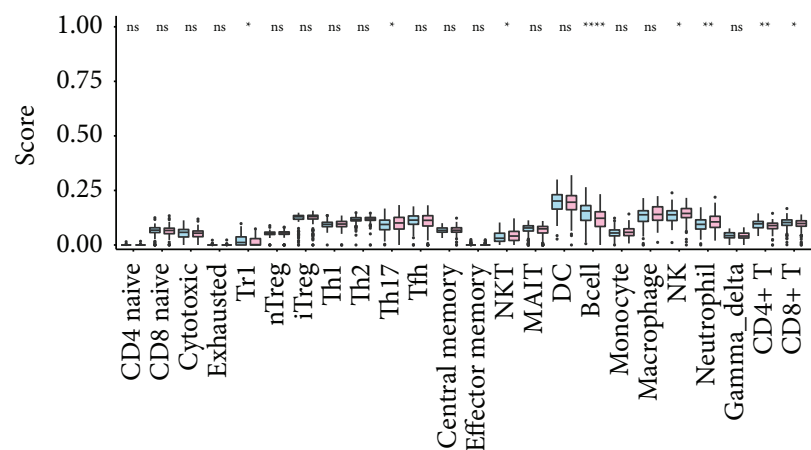

$$
\begin{aligned}
& \text { Group } \\
& \text { 하 low } \\
& \text { ㅂ high }
\end{aligned}
$$

Figure 6: Immune cell abundance analysis between the high-risk and the low-risk group. ${ }^{*} P<0.05,{ }^{* *} P<0.01,{ }^{* * *} P<0.001$, and **** $P<0.0001$.

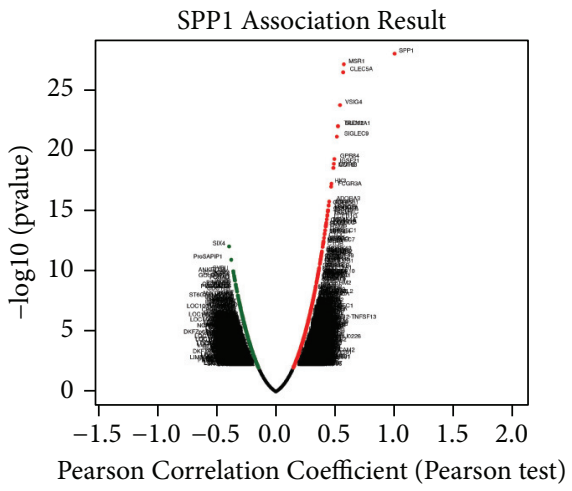

(a)

Figure 7: Continued. 

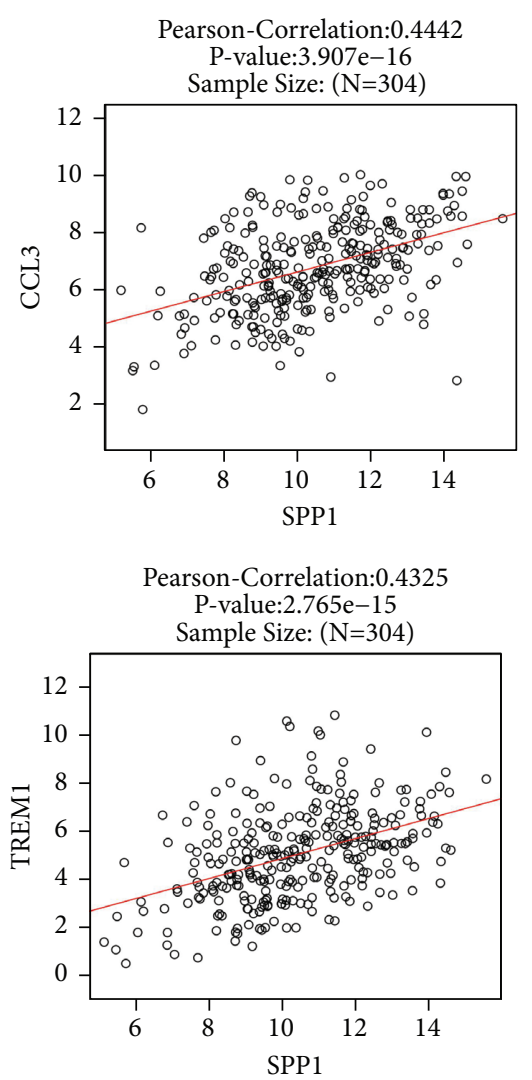
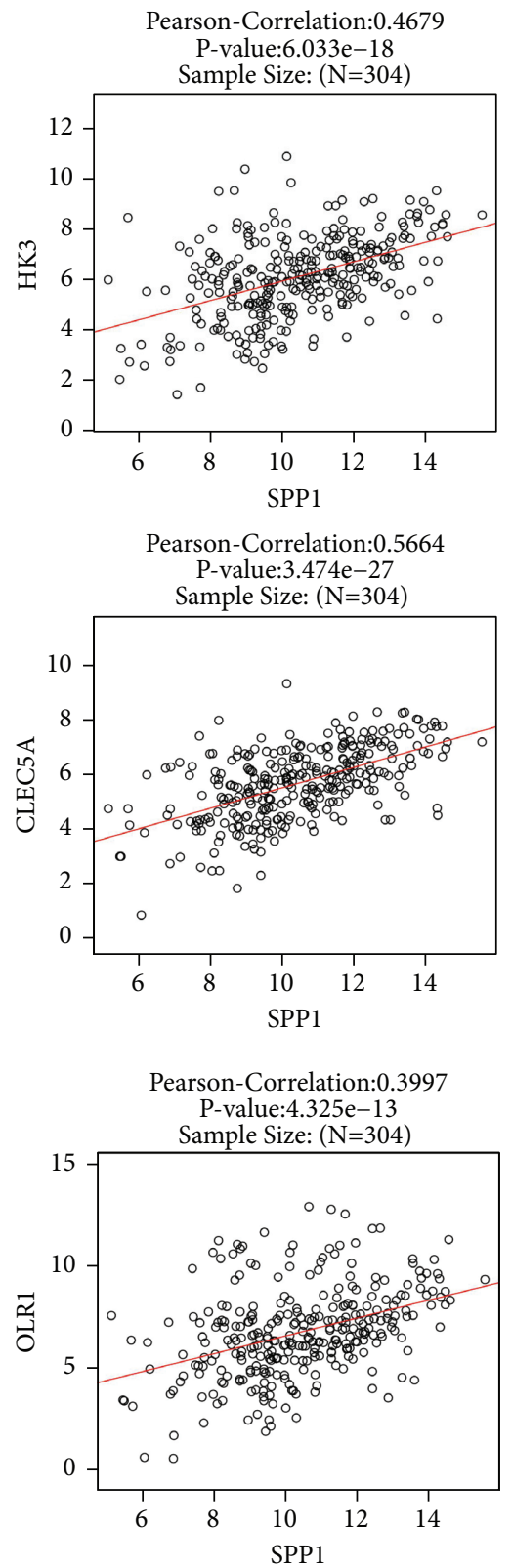

(b)

Figure 7: Continued.
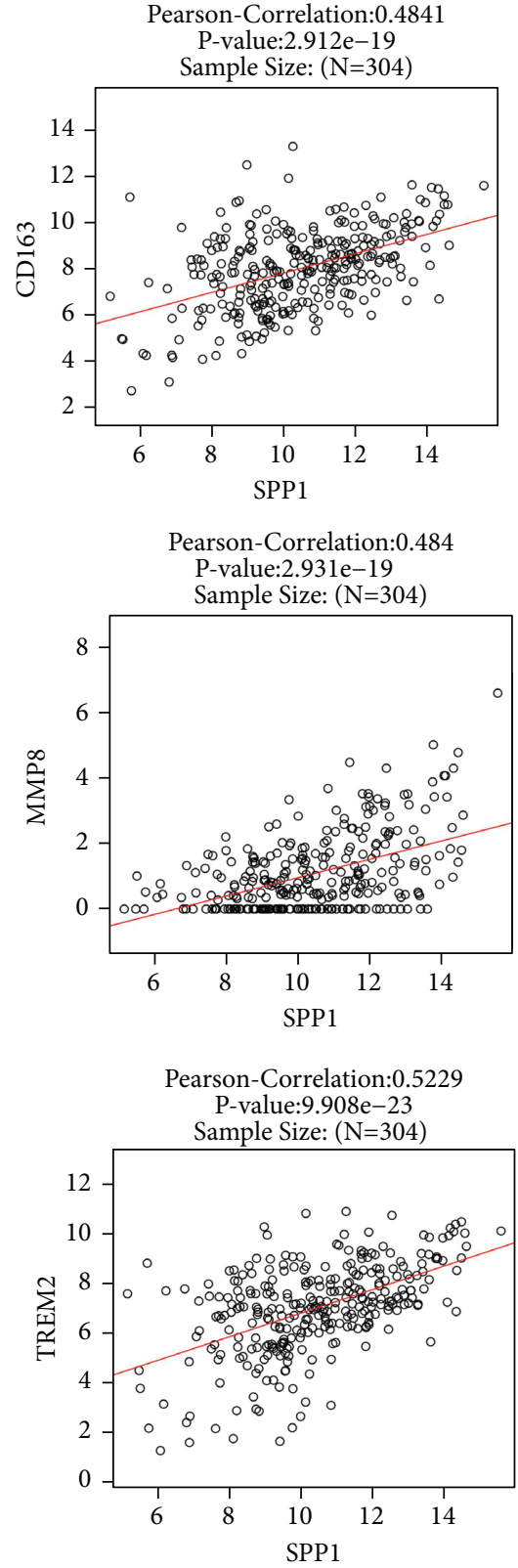

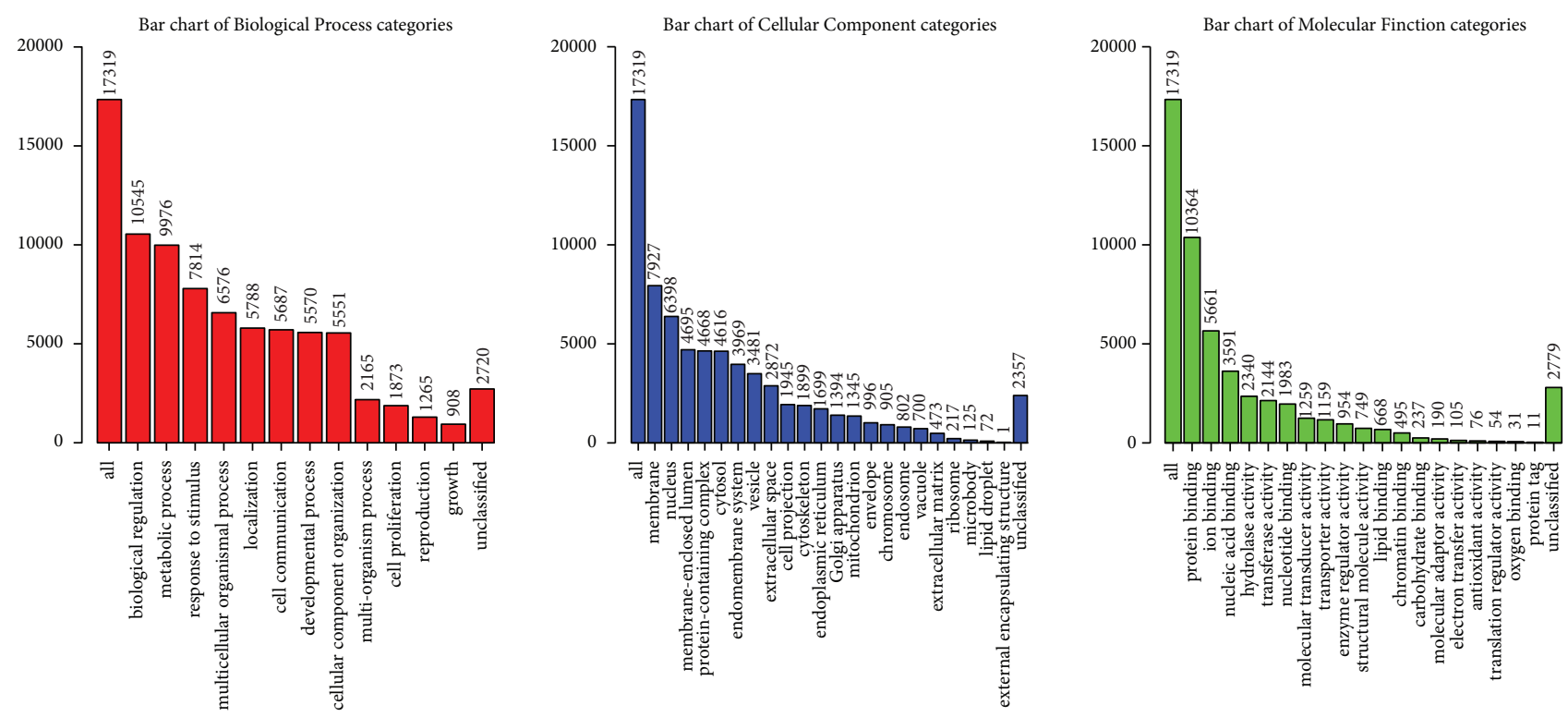

(c)

FIgURE 7: Analysis of SPP1 co-expressed related proteins and Gene Ontology. (a) SPP1 co-expressed related proteins. (b) SPP1 was positively correlated with HK3, CD163, CCL3, CLEC5A, MMP8, TREM1, OLR1, and TREM2. (c) Results of functional enrichment analysis of SPP1 and its co-expressed proteins.

higher proportion of Th17 cells. Increased Th17 cells were associated with clinical stage, lymph node metastasis, and invasion, and therefore accelerate the disease's progression [58]. And this may partially explain the higher level of Th17 cells in the high-risk group.

It has been shown [59] that SPP1 can bind to CD44v6 and promote tumor cell proliferation and survival through JNK pathway. SPP1 also promotes the expression of cancer stem cell markers such as OCT4 and SOX2. This can not only improve the survival rate of cancer cells, but also enhance the resistance to oxaliplatin and other chemotherapy drugs [60]. In colorectal cancer patients, SPP1 induces high expression of CD44v6 through the Wnt- $\beta$-catenin pathway to stimulate cancer progression [61]. This study found that SPP1 is an abnormally expressed gene in the development of cervical cancer. Studies have reported that the expression level of SPP1 is closely related to the occurrence, development, invasion, and metastasis of malignant tumors [62]. In order to further understand the exact mechanism of SPP1 promoting tumor development, this study conducted in-depth bioinformatics analysis on the cancer-promoting mechanism of SPP1. Through the analysis, we found that SPP1 was co-expressed with HK3, CD163, CCL3, CLEC5A, MMP8, TREM1, OLR1, and TREM2.

In colorectal cancer, HK3 overexpression was associated with epithelial-mesenchymal transition [63]. It has been reported that OLR1 promotes pancreatic cancer metastasis via increased c-Myc expression and transcription of HMGA2 [64]. In human osteosarcoma cells, CCL3 promotes angiogenesis by dysregulation of VEGFa [65]. These results further elucidate the carcinogenic mechanism of SPP1 and increased the understanding of SPP1, that is, SPP1 may be a potential key target for the treatment of cervical cancer. However, the molecular mechanism of how it regulates cells in cervical cancer needs to be further studied.
In conclusion, we utilized the public online database to find the related pathways underlying CC development and establish a CC's prognostic model. First, the PI3K-Akt signaling pathway, the Ras signaling pathway, and the viral carcinogenesis pathway may be the critical pathways for CC development. Second, we established a seven-gene prognostic signature for CC and validated the effects of the signature. Finally, we explored the possible mechanism underlying the prognostic signature p53 signaling pathway and further elucidate the carcinogenic mechanism of SPP1.

\section{Data Availability}

Publicly available datasets were analyzed in this study. These can be found in The Cancer Genome Atlas (https://portal. gdc.cancer.gov/) and the NCBI Gene Expression Omnibus (GSE6791, GSE63514, GSE7803, GSE9750, GSE39001, and GSE52903).

\section{Disclosure}

A preprint has previously been published [66].

\section{Conflicts of Interest}

The authors declare that they have no conflicts of interest.

\section{Supplementary Materials}

Supplementary Figure 1: workflow chart of this study. Supplementary Figure 2: quality control of the six datasets. Supplementary Figure 3: KEGG analysis of the top 200 coexpressed genes of the 7 genes of the prognostic signature. KEGG, Kyoto Encyclopedia of Genes and Genomes. Supplementary Table 1: 108 Common DEGs of the six datasets. 
Supplementary Table 2: the associations between overall survival and 108 common DEGs using univariate cox analysis. Supplementary Table 3: the risk score and risk group of each patient of the TCGA database. Supplementary Table 4: gene sets enriched in the high-risk group. Supplementary Table 5: immune cells abundance analysis of the high-risk group and the low-risk group. (Supplementary Materials)

\section{References}

[1] F. Bray, J. Ferlay, I. Soerjomataram, R. L. Siegel, L. A. Torre, and A. Jemal, "Global cancer statistics 2018: GLOBOCAN estimates of incidence and mortality worldwide for 36 cancers in 185 countries," CA: A Cancer Journal for Clinicians, vol. 68, no. 6, pp. 394-424, 2018.

[2] S. Yu, C. S. Yang, J. Li et al., "Cancer prevention research in China," Cancer Prevention Research, vol. 8, no. 8, pp. 662-674, 2015.

[3] X. Jiang, H. Tang, and T. Chen, "Epidemiology of gynecologic cancers in China," Journal of gynecologic oncology, vol. 29, no. 1, Article ID e7, 2018.

[4] W. Chen, R. Zheng, S. Zhang et al., "Cancer incidence and mortality in China in 2013: an analysis based on urbanization level," Chinese Journal of Cancer Research, vol. 29, no. 1, pp. 1-10, 2017.

[5] H. Zur Hausen, "Papillomaviruses and cancer: from basic studies to clinical application," Nature Reviews Cancer, vol. 2, no. 5, pp. 342-350, 2002.

[6] M. Schiffman and N. Wentzensen, "From human papillomavirus to cervical cancer," Obstetrics \& Gynecology, vol. 116, no. 1, pp. 177-185, 2010.

[7] A. I. Ojesina, L. Lichtenstein, S. S. Freeman et al., "Landscape of genomic alterations in cervical carcinomas," Nature, vol. 506, no. 7488, pp. 371-375, 2014.

[8] Cancer Genome Atlas Research Network, Albert Einstein College of Medicine, Analytical Biological Services et al., "Integrated Genomic and Molecular Characterization of Cervical Cancer," Nature, vol. 543, no. 7645, pp. 378-384, 2017.

[9] J. Huang, Z. Qian, Y. Gong et al., "Comprehensive genomic variation profiling of cervical intraepithelial neoplasia and cervical cancer identifies potential targets for cervical cancer early warning," Journal of Medical Genetics, vol. 56, no. 3, pp. 186-194, 2019.

[10] C. Marth, F. Landoni, S. Mahner, M. McCormack, A. Gonzalez-Martin, and N. Colombo, "Cervical cancer: ESMO Clinical Practice Guidelines for diagnosis, treatment and follow-up," Annals of Oncology, vol. 28, no. 4, pp. iv72-iv83, 2017.

[11] H. Zeng, R. Zheng, Y. Guo et al., "Cancer survival in China, 2003-2005: a population-based study," International Journal of Cancer, vol. 136, no. 8, pp. 1921-1930, 2015.

[12] A. Heintz, F. Odicino, P. Maisonneuve et al., "Carcinoma of the fallopian tube," International Journal of Gynecology \& Obstetrics, vol. 95, no. 1, pp. S145-S160, 2006.

[13] S. Junnila, A. Kokkola, T. Mizuguchi et al., "Gene expression analysis identifies over-expression ofCXCL1,SPARC,SPP1, andSULF1in gastric cancer," Genes, Chromosomes and Cancer, vol. 49, no. 1, pp. 28-39, 2010.

[14] E. K. Choe, J. W. Yi, Y. J. Chai, and K. J. Park, "Upregulation of the adipokine genes ADIPOR1 and SPP1 is related to poor survival outcomes in colorectal cancer," Journal of Surgical Oncology, vol. 117, no. 8, pp. 1833-1840, 2018.

[15] M. Zhao, W. Huang, S. Zou, Q. Shen, and X. Zhu, "A fivegenes-based prognostic signature for cervical cancer overall survival prediction," International Journal of Genomics, vol. 2020, Article ID 8347639, 13 pages, 2020.

[16] L. Gautier, L. Cope, B. M. Bolstad, and R. A. Irizarry, "affy-analysis of Affymetrix GeneChip data at the probe level," Bioinformatics, vol. 20, no. 3, pp. 307-315, 2004.

[17] M. E. Ritchie, B. Phipson, D. Wu et al., "Limma powers differential expression analyses for RNA-sequencing and microarray studies," Nucleic Acids Research, vol. 43, no. 7, Article ID e47, 2015.

[18] G. Yu, L.-G. Wang, Y. Han, and Q.-Y. He, "clusterProfiler: an $\mathrm{R}$ package for comparing biological themes among gene clusters," OMICS: A Journal of Integrative Biology, vol. 16, no. 5, pp. 284-287, 2012.

[19] A. Colaprico, T. C. Silva, C. Olsen et al., "TCGAbiolinks: an R/ Bioconductor package for integrative analysis of TCGA data," Nucleic Acids Research, vol. 44, no. 8, Article ID e71, 2016.

[20] J. Liu, T. Lichtenberg, K. A. Hoadley et al., "An integrated TCGA pan-cancer clinical data resource to drive high-quality survival outcome analytics," Cell, vol. 173, no. 2, pp. 400-e11, 2018.

[21] A. Subramanian, P. Tamayo, V. K. Mootha et al., "Gene set enrichment analysis: a knowledge-based approach for interpreting genome-wide expression profiles," Proceedings of the National Academy of Sciences, vol. 102, no. 43, pp. 1554515550, 2005.

[22] Y. R. Miao, Q. Zhang, Q. Lei et al., "ImmuCellAI: a unique method for comprehensive T-cell subsets abundance prediction and its application in cancer immunotherapy," Advanced Science (Weinheim, Baden-Wurttemberg, Germany), vol. 7, no. 7, Article ID 1902880, 2020.

[23] B. Liang, C. Li, and J. Zhao, "Identification of key pathways and genes in colorectal cancer using bioinformatics analysis," Medical Oncology, vol. 33, no. 10, Article ID 111, 2016.

[24] X. Liu, J. Wu, D. Zhang et al., "Identification of potential key genes associated with the pathogenesis and prognosis of gastric cancer based on integrated bioinformatics analysis," Frontiers in Genetics, vol. 9, Article ID 265, 2018.

[25] W. Zhong, H. Hou, T. Liu et al., "Cartilage oligomeric matrix protein promotes epithelial-mesenchymal transition by interacting with transgelin in colorectal cancer," Theranostics, vol. 10, no. 19, pp. 8790-8806, 2020.

[26] H. Du, M. Pang, X. Hou, S. Yuan, and L. Sun, "PLOD2 in cancer research," Biomedicine \& Pharmacotherapy, vol. 90, pp. 670-676, 2017.

[27] F. Xu, J. Zhang, G. Hu, L. Liu, and W. Liang, "Hypoxia and TGF- $\beta 1$ induced PLOD2 expression improve the migration and invasion of cervical cancer cells by promoting epithelialto-mesenchymal transition (EMT) and focal adhesion formation," Cancer Cell International, vol. 17, no. 1, Article ID 54, 2017.

[28] J. Wei, Y. Wang, K. Shi, and Y. Wang, "Identification of core prognosis-related candidate genes in cervical cancer via integrated bioinformatical analysis," BioMed Research International, vol. 2020, Article ID 8959210, 9 pages, 2020.

[29] A. Gupta, D. Nitoiu, D. Brennan-Crispi et al., "Cell cycle- and cancer-associated gene networks activated by Dsg2: evidence of cystatin A deregulation and a potential role in cell-cell adhesion," PLoS One, vol. 10, no. 3, Article ID e0120091, 2015. 
[30] G. Zhou, L. Yang, A. Gray et al., "The role of desmosomes in carcinogenesis," OncoTargets and Therapy, vol. 10, pp. 4059-4063, 2017.

[31] S. Qin, Y. Liao, Q. Du et al., "DSG2 expression is correlated with poor prognosis and promotes early-stage cervical cancer," Cancer Cell International, vol. 20, no. 1, Article ID 206, 2020.

[32] Q. Liu, A. Li, Y. Tian et al., "The CXCL8-CXCR1/2 pathways in cancer," Cytokine \& Growth Factor Reviews, vol. 31, pp. 61-71, 2016.

[33] R. Yan, H. Shuai, X. Luo, X. Wang, and B. Guan, "The clinical and prognostic value of CXCL8 in cervical carcinoma patients: immunohistochemical analysis," Bioscience Reports, vol. 37, 2017.

[34] K. Gou, J. Liu, X. Feng, H. Li, Y. Yuan, and C. Xing, "Expression of minichromosome maintenance proteins (MCM) and cancer prognosis: a meta-analysis," Journal of Cancer, vol. 9, no. 8, pp. 1518-1526, 2018.

[35] N. Murphy, M. Ring, C. C. B. B. Heffron et al., "p16INK4A, CDC6, and MCM5: predictive biomarkers in cervical preinvasive neoplasia and cervical cancer," Journal of Clinical Pathology, vol. 58, no. 5, pp. 525-534, 2005.

[36] L. Dhont, C. Mascaux, and A. Belayew, "The helicase-like transcription factor (HLTF) in cancer: loss of function or oncomorphic conversion of a tumor suppressor?" Cellular and Molecular Life Sciences, vol. 73, no. 1, pp. 129-145, 2016.

[37] K. Hibi, H. Nakayama, Y. Kanyama et al., "Methylation pattern of HLTF gene in digestive tract cancers," International Journal of Cancer, vol. 104, no. 4, pp. 433-436, 2003.

[38] K. Hibi, Y. Kodera, K. Ito, S. Akiyama, and A. Nakao, "Aberrant methylation of HLTF, SOCS-1, and CDH13 genes is shown in colorectal cancers without lymph node metastasis," Diseases of the Colon \& Rectum, vol. 48, no. 6, pp. 1282-1286, 2005.

[39] S. Cho, S. Cinghu, J.-R. Yu, and W.-Y. Park, "Helicase-like transcription factor confers radiation resistance in cervical cancer through enhancing the DNA damage repair capacity," Journal of Cancer Research and Clinical Oncology, vol. 137, no. 4, pp. 629-637, 2011.

[40] B. D. Rowland and D. S. Peeper, "KLF4, p21 and contextdependent opposing forces in cancer," Nature Reviews Cancer, vol. 6, no. 1, pp. 11-23, 2006.

[41] F. Yu, J. Li, H. Chen et al., "Kruppel-like factor 4 (KLF4) is required for maintenance of breast cancer stem cells and for cell migration and invasion," Oncogene, vol. 30, no. 18, pp. 2161-2172, 2011.

[42] D. Wei, M. Kanai, S. Huang, and K. Xie, "Emerging role of KLF4 in human gastrointestinal cancer," Carcinogenesis, vol. 27, no. 1, pp. 23-31, 2006.

[43] Y. Yang, B. G. Goldstein, H.-H. Chao, and J. Katz, "KLF4 and KLF5 regulate proliferation, apoptosis and invasion in Esophageal cancer cells," Cancer Biology \& Therapy, vol. 4, no. 11, pp. 1216-1221, 2005.

[44] W.-T. Yang and P.-S. Zheng, "Krüppel-like factor 4 functions as a tumor suppressor in cervical carcinoma," Cancer, vol. 118, no. 15, pp. 3691-3702, 2012.

[45] W. T. Yang and P. S. Zheng, "Promoter hypermethylation of KLF4 inactivates its tumor suppressor function in cervical carcinogenesis," PLoS One, vol. 9, no. 2, 2014.

[46] W. Meng and M. Takeichi, "Adherens junction: molecular architecture and regulation," Cold Spring Harbor Perspectives in Biology, vol. 1, no. 6, Article ID a002899, 2009.
[47] C. Birchmeier, W. Birchmeier, and B. Brand-Saheri, "Epithelial-mesenchymal transitions in cancer progression," Cells Tissues Organs, vol. 156, no. 3, pp. 217-226, 1996.

[48] J. Fujimoto, S. Ichigo, R. Hirose, H. Sakaguchi, and T. Tamaya, "Expression of E-cadherin and $\alpha$ - and $\beta$-catenin mRNAs in uterine cervical cancers," Tumor Biology, vol. 18, no. 4, pp. 206-212, 1997.

[49] W. Li, Y. Qi, X. Cui et al., "Characteristic of HPV integration in the genome and transcriptome of cervical cancer tissues," BioMed Research International, vol. 2018, Article ID 6242173, 7 pages, 2018.

[50] A. van der Flier and A. Sonnenberg, "Function and interactions of integrins," Cell and Tissue Research, vol. 305, no. 3, pp. 285-298, 2001.

[51] Y. Bao, L. Wang, L. Shi et al., "Transcriptome profiling revealed multiple genes and ECM-receptor interaction pathways that may be associated with breast cancer," Cellular and Molecular Biology Letters, vol. 24, Article ID 38, 2019.

[52] M. K. Andersen, K. Rise, G. F. Giskeødegård et al., "Integrative metabolic and transcriptomic profiling of prostate cancer tissue containing reactive stroma," Scientific Reports, vol. 8, Article ID 14269, 2018.

[53] P. Yan, Y. He, K. Xie, S. Kong, and W. Zhao, "In silico analyses for potential key genes associated with gastric cancer," PeerJ, vol. 6, Article ID e6092, 2018.

[54] K. Wu, Y. Yi, F. Liu, W. Wu, Y. Chen, and W. Zhang, "Identification of key pathways and genes in the progression of cervical cancer using bioinformatics analysis," Oncology Letters, vol. 16, no. 1, pp. 1003-1009, 2018.

[55] M. A. Wozniak, K. Modzelewska, L. Kwong, and P. J. Keely, "Focal adhesion regulation of cell behavior," Biochimica et Biophysica Acta, vol. 1692, no. 2-3, pp. 103-119, 2004.

[56] M. Agochiya, V. G. Brunton, D. W. Owens et al., "Increased dosage and amplification of the focal adhesion kinase gene in human cancer cells," Oncogene, vol. 18, no. 41, pp. 5646-5653, 1999.

[57] J. Wang, Z. Li, A. Gao, Q. Wen, and Y. Sun, “The prognostic landscape of tumor-infiltrating immune cells in cervical cancer," Biomedicine \& Pharmacotherapy, vol. 120, Article ID 109444, 2019.

[58] Y. Zhang, D. Ma, Y. Zhang et al., "The imbalance of Th17/Treg in patients with uterine cervical cancer," Clinica chimica acta; international journal of clinical chemistry, vol. 412, no. 11-12, pp. 894-900, 2011.

[59] G. Rao, H. Wang, B. Li et al., "Reciprocal interactions between tumor-associated macrophages and CD44-positive cancer cells via osteopontin/CD44 promote tumorigenicity in colorectal cancer," Clinical Cancer Research, vol. 19, no. 4, pp. 785-797, 2013.

[60] L. Ng, T. Wan, A. Chow et al., "Osteopontin overexpression induced tumor progression and chemoresistance to oxaliplatin through induction of stem-like properties in human colorectal cancer," Stem Cells International, vol. 2015, Article ID 247892, 8 pages, 2015.

[61] M. Todaro, M. Gaggianesi, V. Catalano et al., "CD44v6 is a marker of constitutive and reprogrammed cancer stem cells driving colon cancer metastasis," Cell Stem Cell, vol. 14, no. 3, pp. 342-356, 2014.

[62] R. Wei, J. P. C. Wong, and H. F. Kwok, "Osteopontin-a promising biomarker for cancer therapy," Journal of Cancer, vol. 8, no. 12, pp. 2173-2183, 2017.

[63] E. A. Pudova, A. V. Kudryavtseva, M. S. Fedorova et al., "HK3 overexpression associated with epithelial-mesenchymal 
transition in colorectal cancer," BMC Genomics, vol. 19, no. 3, pp. 113-13, 2018.

[64] G. Yang, G. Xiong, M. Feng et al., "OLR1 promotes pancreatic cancer metastasis via increased c-Myc expression and transcription of HMGA2," Molecular Cancer Research, vol. 18, no. 5, pp. 685-697, 2020.

[65] Y. Y. Liao, H. C. Tsai, P. Y. Chou et al., "CCL3 promotes angiogenesis by dysregulation of miR-374b/VEGF-A axis in human osteosarcoma cells," Oncotarget, vol. 7, no. 4, pp. $4310-25,2016$.

[66] S. Meng, R. Ning, J. Zhang et al., "Identification of key pathways and establishment of a seven-gene prognostic signature in cervical cancer," Research Square, 2021. 